\title{
Structure and Dynamics of Dense Monolayers of NO Adsorbed on Rh(111) in Equilibrium with the Gas Phase in the Torr Pressure Range
}

\author{
Keith B. Rider, ${ }^{*}$ Kevin S. Hwang, ${ }^{*}$ Miquel Salmeron ${ }^{6}$ and Gabor A. Somorjai ${ }^{*}$ \\ Materials Sciences Division, Lawrence Berkeley National Laboratory \\ University of California, Berkeley, California 94720
}

\begin{abstract}
Using Scanning Tunneling Microscopy, we show the phase transition between new structures of $\mathrm{NO}$ on $\mathrm{Rh}(111)$ in equilibrium with the gas phase near $300 \mathrm{~K}$, in the Torr pressure range. Two phases with $(2 \times 2)$ and $(3 \times 3)$ periodicity transform into each other as the pressure and temperature change around the equilibrium $P$ - $T$ line. By measuring $P$ and $T$ at coexistence, we determined the heat of adsorption in the $(3 \times 3)$ structure. From the phase boundary dynamics, the activation energy barrier between phases was estimated. The results demonstrate that unique information can be obtained from high-pressure and high-temperature studies.
\end{abstract}

PACS numbers: 68.37.Ef, 82.45.Ju, 68.18.Jk

\footnotetext{
*Also in the Department of Chemistry, University of California, Berkeley, California 94720

${ }^{\dagger}$ Corresponding author. E-mail: salmeron@stm.lbl.gov
} 
Our understanding of the elementary steps of surface reactions comes primarily from experiments performed under low-pressure $\left(10^{-4}-10^{-10}\right.$ Torr $)$ conditions. The motivation for studying gas-surface reactions is the industrial importance of heterogeneous catalysis. The difference between traditional low-pressure studies and industrial applications, which are normally carried out at high pressure, is known as the pressure-gap. In addition, the sample temperature in a low-pressure experiment must be very low if the coverage is to remain the same as that obtained at high pressures. This means that in the divide between traditional surface science and its industrial motivation, there is also a temperature-gap. Bridging these gaps is critical for a fundamental understanding of catalysis. Although the high surface coverage characteristic of high pressures can be achieved at low pressure by lowering the temperature, the structures formed might not be the same, as we have shown recently in the case of $\operatorname{Pt}(111)$ in 200 Torr of $\mathrm{CO}$ [1] At high pressure and temperature, the structures formed are in equilibrium with the gas phase, while at low temperature the structures must be kinetically frozen. The low temperature is also likely to prevent the formation of structures that are reached through an activated process such as surface reconstruction. These problems have been overcome by the development of a high-pressure, hightemperature Scanning Tunneling Microscope (STM). The STM, from RHK Technology, is housed in a reactor chamber connected to a standard ultra-high vacuum apparatus, in which the samples are prepared and characterized. After transferring the sample to the reactor chamber, valves are closed and gases are introduced to a high pressure. The capabilities of the instrument are described elsewhere [2[⿰阝⿹丁口⿹丁口 
Here, we report the discovery of new structures of $\mathrm{NO}$ on $\mathrm{Rh}(111)$ formed at pressures in the Torr range and their transformation dynamics. Two ordered structures are found with $(2 \times 2)$ and $(3 \times 3)$ periodicity relative to the substrate, which are only slightly different in density. Regions of the surface transform from one structure to the other with small changes of temperature and pressure around a phase equilibrium line. The heat of adsorption of molecules in the $(3 \times 3)$ structure was determined using the two-dimensional analog of the Clausius-Clapeyron equation.

A $(2 \times 2)$ structure with coverage of $0.75 \mathrm{ML}$ is known to form either by large $(\sim 20$ Langmuir) exposures to NO at $200 \mathrm{~K}$, or by small ( 2 Langmuir) exposures at $40 \mathrm{~K}$, followed by annealing to $200 \mathrm{~K}$. At room temperature, it is possible to maintain this structure in a background of $10^{-8}$ Torr. X-ray photoelectron diffraction [4] and tensor LEED [5] studies indicate that the unit cell contains three NO molecules, one on top and two in three-fold hollow sites. The on-top molecule sits $0.4 \AA$ higher than those on hollow sites.

The sample was cleaned by $\mathrm{Ar}^{+}$sputtering and annealing in $\mathrm{O}_{2}$. Auger spectroscopy revealed that carbon, the most common contaminant, made up less than $5 \%$ of the surface. After cleaning, the sample was moved to the high-pressure chamber and imaged by STM using etched tungsten tips. In the range of $10^{-8}$ to 0.01 Torr of NO at room temperature, the images show the $(2 \times 2)$ periodicity, with only one maximum per unit cell. The structure is similar to that formed by $\mathrm{CO}$ on $\mathrm{Rh}(111)$ and $\mathrm{Pd}(111)$. STM studies of these structures also show one maximum per unit cell [6]. According to theoretical calculations, the maximum corresponds to the molecule in the top site [0]. It is likely that the same is true for the $(2 \times 2)-3 \mathrm{NO}$ structure. 
Next, we increased the NO pressure at a rate of approximately $0.001 \mathrm{Torr} /$ minute at $300 \mathrm{~K}$. As the pressure entered the range between 0.01 and 0.05 Torr, areas of the surface were seen covered with a $(3 \times 3)$ pattern. These areas grow over the course of a few hours until the entire surface is covered by the $(3 \times 3)$ structure. Fig. 1 shows a series of images taken 55 seconds apart on the same area at 0.03 Torr. In Fig. 1a, the majority of the surface is covered with the $(2 \times 2)$ pattern, with one corner showing a small area of (3×3). The boundary (white line) propagates at a rate of about $20 \AA /$ minute from the upper-right corner to the lower-left corner. In Figs. 1a and 1b, an immobile defect has been marked for reference.

In Fig. 2, we show an island of $(3 \times 3)$ (inside the dotted line) surrounded by areas of $(2 \times 2)$ structure. Two straight lines have been drawn that separate two unit cells of $(3 \times 3)$ or three unit cells of $(2 \times 2)$. The cursor profile along A-B (shown at the bottom) reveals two differences between these structures: (1) The corrugation of the $(3 \times 3)$ is always higher, and (2) the apparent height is also higher in the $(3 \times 3)$ regions. In the present image, the corrugation in the $(3 \times 3)$ region is $0.2 \AA$ on average, compared with the $0.1 \AA$ corrugation of the $(2 \times 2)$ region. These values vary by a factor of two from image to image, as a result of different tunneling conditions and tip structure. On average, the $(3 \times 3)$ to $(2 \times 2)$ corrugation ratio is 4 to 1 . Also in the figure, the apparent height of the (3×3) regions is $\sim 0.1 \AA$ higher than that of the $(2 \times 2)$ regions. In general, this apparent height difference varies between 0.1 and $0.5 \AA$, depending on tunneling conditions and tip structure. The higher corrugation of the $(3 \times 3)$ structure could be the result of the larger dimensions of the unit cell, which causes the top-site NO molecules to be farther apart and allows the tip to better follow the molecular contours. It also could be the result 
of restructuring of the rhodium substrate. The higher elevation of the base line in the denser structure might indicate an expansion of the top layer of rhodium atoms. The answer to these questions requires experiments with other techniques, such as X-ray diffraction.

While the $(2 \times 2)$ structure has been solved by LEED, no information is available concerning the $(3 \times 3)$. The models shown in the middle of Fig. 2 are proposed based on the following considerations. First, the coverage in the $(3 \times 3)$ structure can be only slightly higher than the $0.75 \mathrm{ML}$ coverage of the $(2 \times 2)$, because this is already very dense (only $3.16 \AA$ separates adjacent molecules). Second, the STM images show that the rows of maxima in both structures are parallel and are separated by multiples of the $\mathrm{Rh}-\mathrm{Rh}$ distance. This indicates that the molecules producing the maxima occupy similar on-top sites in both structures. A $(3 \times 3)$ structure satisfying these two conditions and preserving the hexagonal packing of $\mathrm{NO}$ in the $(2 \times 2)$ can be obtained by a rigid rotation of the $\mathrm{NO}$ layer of $10.9^{\circ}$, followed by a linear compression of $1.8 \%$. A more symmetric structure with NO molecules in 3-fold hollow sites can be obtained by a small relaxation of the molecules inside the cell. In both of these models, the surface coverage is $0.778 \mathrm{ML}$, only $3.7 \%$ larger than the $(2 \times 2)-3 \mathrm{NO}$ coverage.

Another important observation is the facile displacement of the boundary between the two NO structures, indicative of dynamic equilibrium. Fig. 3 illustrates this phenomenon more dramatically. A series of $500 \AA \times 500 \AA$ images were taken at 0.01 Torr. Although the large size of the images does not allow the individual cells to be visible, higher resolution images show that the periodicity in the bright areas is $(3 \times 3)$ and that in the dark areas $(2 \times 2)$. Fig. 3a shows several $(3 \times 3)$ domains, labeled 1, 2 and 3 . 
Initially, domains 1 and 2 are part of a larger domain. In Figs. 3b-e, this domain splits in half and domain 1 dissipates. In Fig. 3f, domains 1 and 2 have dissipated, but domain 3 remains. In the next image (not shown), domain 3 has also dissipated, leaving a surface entirely covered by the $(2 \times 2)$ structure. The formation of $(3 \times 3)$ domains is not affected by the presence of the tip, since similar phenomena are observed whether the gas is added with the tip far from the surface or within tunneling range. The facile displacement of the boundary between the two structures, which occurs on a time scale of seconds, indicates that they are energetically separated by a barrier of approximately $0.7 \mathrm{eV}[8]$, so that thermal fluctuations convert small areas of the surface from one structure to the other. This barrier, of course, is not the energy difference between the two structures, as we will discuss next.

Until now, we have described experiments performed at room temperature. To study the phase equilibrium and the energy difference between the two structures, experiments were performed at temperatures between $300 \mathrm{~K}$ and $350 \mathrm{~K}$. As expected, the transition pressure $P$ increases as a function of temperature $T$. Fig. 4 is a plot of $\ln (P)$ vs. $1 / T$, each point representing a pressure and temperature in which both $(2 \times 2)$ and $(3 \times 3)$ structures were observed on the surface. In the region close to the line, both structures are visible, even after long periods of time (more than 12 hours). In the region above the line, only the $(3 \times 3)$ structure is observed; below the line only the $(2 \times 2)$ structure is observed. This line represents the equilibrium condition since it could be reached either by increasing or decreasing the pressure without hysteresis. The slope of the straight line is $1.1 \pm 0.2 \mathrm{eV} / \mathrm{k}(\mathrm{k}=$ Boltzman constant $)$ 
The state of the gas-surface system is described by the three variables $P, T$ and $\varphi$, where $\varphi$ is the two-dimensional surface pressure. When the system consists of a gas phase and two surface phases ( 1 and 2 , for the $(2 \times 2)$ and $(3 \times 3)$ phases, respectively), the equilibrium condition $\mu(P, T)=\mu_{1}(P, T, \varphi)=\mu_{2}(P, T, \varphi)$ places two constraints on $P, T$ and $\varphi$, leaving one degree of freedom. It is this degree of freedom that is represented by the line in Fig. 4. According to Hill [9], when a perfect gas is in equilibrium with two surface phases

$$
\frac{d \ln P}{d T}=\frac{\Gamma_{2}\left(H_{2}-H_{g}\right)-\Gamma_{1}\left(H_{1}-H_{g}\right)}{k T^{2}\left(\Gamma_{1}-\Gamma_{2}\right)},
$$

where $H_{i}$ is the molar enthalpy and $\Gamma_{\mathrm{i}}$ the coverage for surface phase $i$. Recognizing that $\left(H_{1}-H_{g}\right)$ and $\left(H_{2}-H_{g}\right)$ are heats of adsorption, which we will call $h_{1}$ and $h_{2}$,

$$
\frac{d \ln P}{d(1 / T)}=\frac{\frac{\Gamma_{1}}{\Gamma_{2}} h_{1}-h_{2}}{k\left(\Gamma_{1} / \Gamma_{2}-1\right)} .
$$

The heat of adsorption for $\mathrm{Rh}(111)$ surfaces saturated with $\mathrm{NO}$ in low-pressure conditions has been measured to be $1.0 \pm 0.1 \mathrm{eV}[10]$. Under these conditions, the surface forms the $(2 \times 2)-3 \mathrm{NO}$ structure, so $h_{1}=1.0 \mathrm{eV}$. From the slope in Fig. 4 and given that $\Gamma_{2}=0.778$ and $\Gamma_{1}=0.75$, we calculate the heat of adsorption for the $(3 \times 3)-7 \mathrm{NO}$ structure to be $0.9 \pm$ $0.1 \mathrm{eV}$. The difference in the heat of adsorption for the two structures is therefore on the order of $0.1 \mathrm{eV}$, a result anticipated from the observation that the two phases can occupy similar areas of the surface.

The novel observation of molecularly resolved surface phenomena at high pressure and temperature heralds the beginning of a new era in surface science. By bridging the pressure and temperature gaps with high-pressure, high-temperature STM, 
we have the opportunity to study molecular level structures and processes at gas-surface interfaces under conditions that were previously inaccessible. We have shown the transition from the $(2 \times 2)-3 \mathrm{NO}$ structure, which is known from low-pressure surface science experiments, to a new $(3 \times 3)$ structure, which only forms in equilibrium with the gas phase at high pressure. By directly observing the transition between these structures at several temperatures and pressures, we could measure the heat of adsorption in the new structure to be $0.9 \pm 0.1 \mathrm{eV}$, and from the dynamic behavior of the phase boundary, we deduced an energy barrier of approximately $0.7 \mathrm{eV}$. These are the types of studies that are most relevant for a molecular level understanding of surface-catalyzed reactions.

This work is supported by the Director, Office of Energy Research, Office of Basic Energy Sciences, Material Sciences Division, of the U. S. Department of Energy under contract No. DE-AC03-76F00098. 


\section{References}

[1] J. A. Jensen, K. B. Rider, M. Salmeron, and G. A. Somorjai, Phys. Rev. Lett. 80, 1228 (1998).

[2] J. A. Jensen, K. B. Rider, Y. Chen, M. Salmeron, and G. A. Somorjai, J. Vac. Sci. Technol. B 17, 1080 (1999).

[3] B. J. McIntyre, M. Salmeron, and G. A. Somorjai, Rev. Sci. Instrum. 64, 687 (1993).

[4] Y. J. Kim et al., Surf. Sci. 359, 269 (1996).

[5] I. Zasada, M. A. Van Hove, and G. A. Somorjai, Surf. Sci. Lett. 418, L89 (1998).

[6] M. Rose and M. Salmeron, unpublished data.

[7] M. Rose, M. Salmeron, and P. Sautet, unpublished data.

[8] This energy barrier is estimated using $t(1 \mathrm{sec})=\tau \exp (E / k T)$, with $\tau=10^{-13} \mathrm{sec}$. A change of this value by an order of magnitude changes the value of $E$ by $7 \%$.

[9] T. L. Hill, J. Chem. Phys. 17, 520 (1949).

[10] H. J. Borg, J. F. C.-J. M. Reijerse, R. A. van Santen, and J. W. Niemantsverdriet, J. Chem. Phys. 101, 10052 (1994). 


\section{Figure Captions}

FIG. 1. STM images of $\mathrm{Rh}(111)$ in 0.03 Torr of $\mathrm{NO}$ at $25^{\circ} \mathrm{C}$, showing the phase transition between a $(2 \times 2)$ and a $(3 \times 3)$ structure. The $100 \AA \times 100 \AA$ images were obtained at 55second intervals $(I=440 \mathrm{pA}, \mathrm{V}=99 \mathrm{mV})$. In (a), the majority of the surface shows the $(2 \times 2)$ structure, except for the upper right-hand corner, which shows the $(3 \times 3)$ structure. In (b) and (c), the domain boundary between the two phases moves across the image at a rate of $\sim 20 \AA /$ minute. (a) and (b) share a common defect, marked with a circle. The closepacked rows of both patterns are aligned, and the maxima are separated by multiples of the Rh-Rh distance. This indicates that the bright spots correspond to NO molecules on similar sites in both the $(2 \times 2)$ and the $(3 \times 3)$ structures.

FIG. 2. A $200 \AA \times 200 \AA \mathrm{STM}$ image taken in 0.03 Torr $\mathrm{NO}$ at $25^{\circ} \mathrm{C}$, showing a $(3 \times 3)$ domain surrounded by the $(2 \times 2)$ structure. It is known that the $(2 \times 2)-3 \mathrm{NO}$ structure contains one top-site NO molecule and two molecules on hollow sites. Two similar models are proposed for the $(3 \times 3)$ structure. The first (left) consists of one top-site NO molecule and six molecules near hollow sites. In the second model (right), the near hollow-site molecules are relaxed so that they occupy 3-fold hollow sites. In both models the coverage is $0.778 \mathrm{ML}$ and are consistent with the STM images. A line profile taken from line A-B on the image is shown. Note the higher corrugation in the $(3 \times 3)$ domain and its higher apparent height, $\sim 0.1 \AA$ above that of the $(2 \times 2)$ domain. 
FIG. 3. Sequence of $500 \AA \times 500 \AA \mathrm{STM}$ images in $0.01 \mathrm{Torr} \mathrm{NO}$ at $25^{\circ} \mathrm{C}$, showing the evolution of domains of the $(3 \times 3)$ structure $(I=214 \mathrm{pA}, \mathrm{V}=101 \mathrm{mV})$. The brighter areas correspond to the $(3 \times 3)$ structure and the darker background to the $(2 \times 2)$. The images were taken 55 seconds apart. In (a), several ( $3 \times 3)$ domains can be seen, labeled 1, 2 and 3. Initially, 1 and 2 are part of a larger domain. In (b)-(e), this domain splits in half and domain 1 dissipates. In (f), 1 and 2 have dissipated, but 3 remains. In the next image, domain 3 has also dissipated, leaving a surface entirely covered by the $(2 \times 2)$ structure (not shown).

FIG. 4. Plot of $\ln (P)$ vs. $1 / T$ for values where the $(2 \times 2)$ to $(3 \times 3)$ transition takes place. The line through the experimental points separates regions where the $(2 \times 2)$ structure is stable (below) and where the ( $3 \times 3)$ structure is stable (above). The slope of this line is 1.1 $\pm 0.2 \mathrm{eV} / \mathrm{k}$. 